\title{
A parametric study of droplet deformation through a microfluidic contraction
}

\section{J. E. Harvie* M. R. Davidson ${ }^{\dagger} \quad$ J. J. Cooper-White ${ }^{\ddagger}$ M. Rudman ${ }^{\S}$}

(Received 29 November 2004, revised 18 February 2005)

\begin{abstract}
A numerical parametric study of droplet deformation within an axisymmetric micro-fluidic contraction is performed. The simulations use a transient Volume of Fluid finite volume algorithm and cover parameter ranges representative of micro-sized liquid-liquid systems. We consider two disperse continuous viscosity ratios. When the phases have equal viscosities, the predicted droplet shapes range from short 'slugs' constrained by the contraction walls through to long thin 'filaments'. When the disperse phase viscosity is lower than that of the continuous phase, capillary waves and other instabilities develop along
\end{abstract}

${ }^{*}$ Dept. Chemical \& Biomolecular Engineering, University of Melbourne, Australia. mailto:daltonh@unimelb.edu. au

${ }^{\dagger}$ Dept. Chemical \& Biomolecular Engineering, University of Melbourne, Australia.

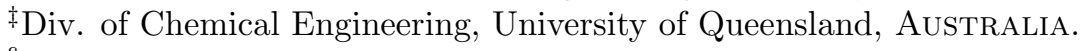

$\S$ CSIRO, Manufacturing \& Infrastructure Technology, Melbourne, Australia.

See http://anziamj.austms.org.au/V46/CTAC2004/Har2 for this article, (C) Austral. Mathematical Soc. 2005. Published April 19, 2005. ISSN 1446-8735 
the droplet surface, leading to more complex shape development and associated fluid dynamics.

\section{Contents}

1 Introduction

2 Problem description

3 Simulation method

4 Results: equal viscosities, $\mu_{\mathrm{d}}=1$

5 Results: lower viscosity droplet, $\mu_{\mathrm{d}}=0.1$

6 Conclusions

\section{Introduction}

The deformation of a droplet as it passes through a microfluidic contraction is a fundamental flow problem having relevance to a broad range of Micro-Electro-Mechanical Systems (MEMS) applications. A characteristic of microfluidic flows is that surface forces assume a greater importance than in conventionally scaled flows. For flows involving more than one liquid, this implies that capillary effects are more dominant than in conventional flows, and this presents new challenges when modeling such systems.

There have been few experimental studies concerned with immiscible fluids passing through micro-fluidic contractions. An exception is the work of 
Anna, Bontoux \& Stone [1] who used a micro-fluidic contraction as a 'flow focusing' device to break a stream of disperse phase fluid into droplets. The deformation of larger, millimetre sized droplets moving through contractions has been studied experimentally by several researchers, including Han \& Funatsu [4]. Numerical investigations of droplets moving through contractions have generally employed the creeping flow approximation; however, Whyte et al. [12] used the full Navier-Stokes equations when simulating droplet deformation through millimetre sized contractions. Droplet deformation in general extensional and shear flows has been extensively studied both experimentally and numerically. Eggers [3] and Stone [9] give good reviews of this topic.

In this study we analyse and discuss the effect that the Reynolds number, surface tension strength and the disperse continuous phase viscosity ratio have on the deformation of a droplet as it passes through an axisymmetric contraction. We consider parameter ranges that are relevant to liquid-liquid systems (for example, water in oil) and characteristic of MEMS applications, and perform the simulations using a transient Volume of Fluid (VOF) finite volume algorithm.

\section{Problem description}

As shown in Figure 1, the problem under consideration consists of a droplet entrained in a continuous liquid phase passing through a $4: 1$ axisymmetric contraction. All lengths are non-dimensionalised by the radius of the inlet $R^{*}$ so that the contraction radius is $1 / 4$. The contraction length is set to 5 and the initial droplet diameter to 1 .

Three equations describe motion throughout the disperse (that is, droplet) and continuous phases: a continuity equation, a volume-averaged incompressible Navier-Stokes momentum equation, and an advection equation which 


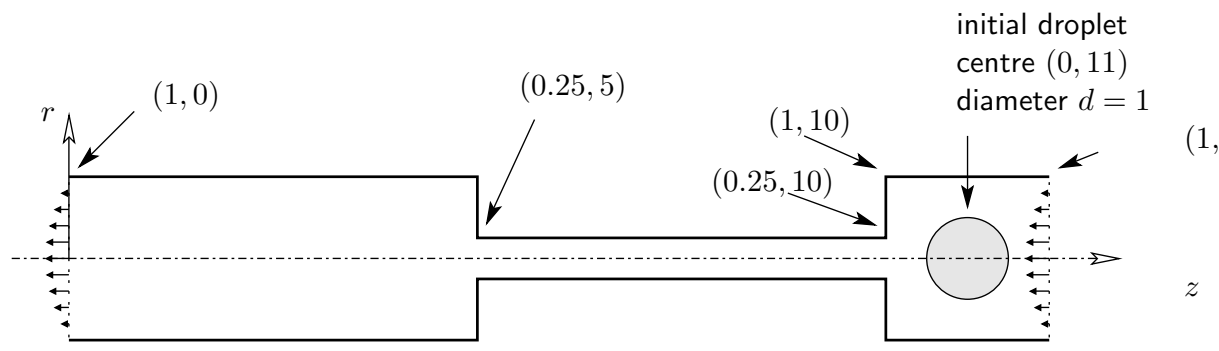

FiguRE 1: The geometry used in the computational problem. All lengths are normalised by the inlet radius $R^{*}$ and cylindrical coordinates $(r, z)$ are used.

describes the evolution of the disperse phase volume fraction $\phi$;

$$
\begin{aligned}
& \boldsymbol{\nabla} \cdot \boldsymbol{u}=0, \\
& \frac{\partial \rho \boldsymbol{u}}{\partial t}+\boldsymbol{\nabla} \cdot \rho \boldsymbol{u} \boldsymbol{u}=-\boldsymbol{\nabla} p+\frac{1}{\mathrm{We}} \kappa \delta(\boldsymbol{x}) \boldsymbol{n}+\frac{1}{\mathrm{Re}} \boldsymbol{\nabla} \cdot \mu\left[\boldsymbol{\nabla} \boldsymbol{u}+(\boldsymbol{\nabla} \boldsymbol{u})^{\top}\right], \\
& \frac{\partial \phi}{\partial t}+\boldsymbol{\nabla} \cdot \phi \boldsymbol{u}=0 .
\end{aligned}
$$

All three equations are employed in a non-dimensional form. The velocity is scaled by the average inlet velocity $v^{*}$, density by the continuous phase density $\rho_{\mathrm{c}}^{*}$, and viscosity by the continuous phase viscosity $\mu_{\mathrm{c}}^{*}$. An asterix denotes a physical quantity.

The second term on the right of equation (2) is a surface tension induced stress jump which occurs at the disperse continuous phase interface. In this term $\kappa$ is the signed local curvature of the interface, $\delta(\boldsymbol{x})$ is the Dirac delta function, non-zero only on the interface, and $\boldsymbol{n}$ is a unit vector directed normal to the interface and into the disperse phase. As equation (2) is applied over both phases, the viscosity $\mu$ is a function of the local volume fraction $\phi$, and varies between 1 and the disperse continuous phase viscosity ratio, $\mu_{\mathrm{d}}=$ $\mu_{\mathrm{d}}^{*} / \mu_{\mathrm{c}}^{*}$. Two different viscosity ratios are considered in the simulations, $\mu_{\mathrm{d}}=$ 0.1 and 1 . The density is set to be equal in both phases, and gravitational forces are neglected as their effect in liquid-liquid micro-sized flows is small. 
In this study the behaviour of the droplet as it passes through the contraction is determined by the magnitude of three forces - inertial, viscous and surface tension. Ratios between these forces are given by the Reynolds (Re), Weber (We) and capillary (Ca) numbers, defined by

$$
\mathrm{Re}=\frac{\rho_{\mathrm{c}}^{*} v^{*} R^{*}}{\mu_{\mathrm{c}}^{*}}, \quad \mathrm{We}=\frac{\rho_{\mathrm{c}}^{*} v^{* 2} R^{*}}{\sigma^{*}} \quad \text { and } \quad \mathrm{Ca}=\frac{\mathrm{We}}{\mathrm{Re}}=\frac{v^{*} \mu_{\mathrm{c}}^{*}}{\sigma^{*}},
$$

respectively. An order of magnitude analysis on equation (2) shows that the magnitude of surface tension forces relative to both viscous and inertial forces can be measured via

$$
S=\frac{1}{\mathrm{We}+\mathrm{Ca}}=\frac{\sigma^{*}}{v^{*} \mu_{\mathrm{c}}^{*}+\rho_{\mathrm{c}}^{*} v^{* 2} R^{*}} .
$$

As a balance between three forces determines droplet behaviour, only two of the above non-dimensional numbers (along with $\mu_{\mathrm{d}}$ ) are required to classify a particular flow regime. In discussing the simulation results we use Re and $S$ - Re specifies the ratio of inertial to viscous forces acting in the flow, while $S$ specifies the magnitude of surface tension present, relative to the other forces combined.

\section{Simulation method}

The simulations use a finite volume code due to Rudman [8], adapted to model shear-thinning fluids. This code has been successfully used to model the formation and subsequent 'pinch-off' of both Newtonian and non-Newtonian pendant drops [2] and the deformation of Newtonian droplets through millimeter sized contractions [12]. The Volume of Fluid (VOF) technique tracks the disperse continuous phase interface, and surface tension forces are applied using a variation of the Continuum Surface Force (CSF) model. The voF function is advected using the Youngs scheme [13]. The domain is discretised on a uniform, staggered mesh. 
All of the simulations presented here use a mesh of dimensions $64 \times 768$. Using a finer mesh did not significantly alter droplet deformation behaviour. We assume the fluid entering the domain has a fully developed Poiseuille profile; tests show that droplet behaviour within the contraction is quite insensitive to the form of this profile. At the domain exit the pressure gradient normal to the outlet port is chosen to ensure global mass conservation. All domain boundaries are non-slip and non-wetting with respect to the disperse phase liquid. Non-wetting boundaries are used as a thin film of continuous phase fluid usually separates the disperse phase fluid from any walls in microfluidic experiments [1, for example]. Each simulation is performed on a single $2.4 \mathrm{GHz}$ Xeon processor. Due to constraints on viscous and capillary time steps, a complete run requires up to several weeks to complete.

\section{Results: equal viscosities, $\mu_{\mathrm{d}}=1$}

A series of droplet deformation simulations was conducted with $\mu_{\mathrm{d}}=1$. Some images from these simulations are shown in Figure 2, along with the simulation locations on a $\mathrm{Ca}$ and We phase chart. The phase chart also shows lines of constant Re and $S$. The Re lines show that viscous forces dominate inertial forces in the top left corner of the chart while inertial forces dominate viscous forces in the bottom right. The $S$ lines show that surface tension forces are weak in the top right corner and strong in the bottom left.

The simulations were conducted at the intersection points between lines of constant Ohrnesorge number $\left(\mathrm{Oh}=\sqrt{\mathrm{We}} / \mathrm{Re}=\mu_{\mathrm{c}}^{*} / \sqrt{\rho_{\mathrm{c}}^{*} \sigma^{*} R^{*}}\right)$ and lines of constant We. The Ohrnesorge number is a function only of the droplet dimensions, surface tension coefficient and continuous phase properties. If these parameters are all constant, then We (or Re) can be regarded as a nondimensional velocity. Thus, simulations conducted at various values of We (or $\mathrm{Re}$ ) along a line of constant $\mathrm{Oh}$ are representative of experiments done with the same experimental setup, but with a variety of continuous phase 

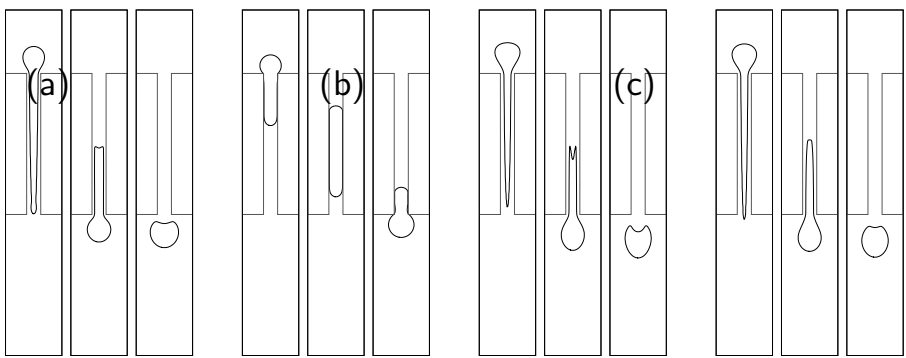

(d)
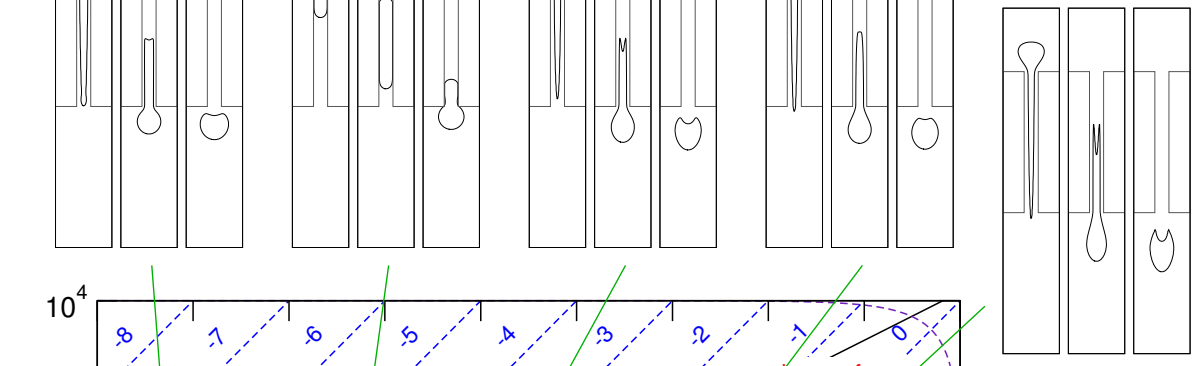

(e)
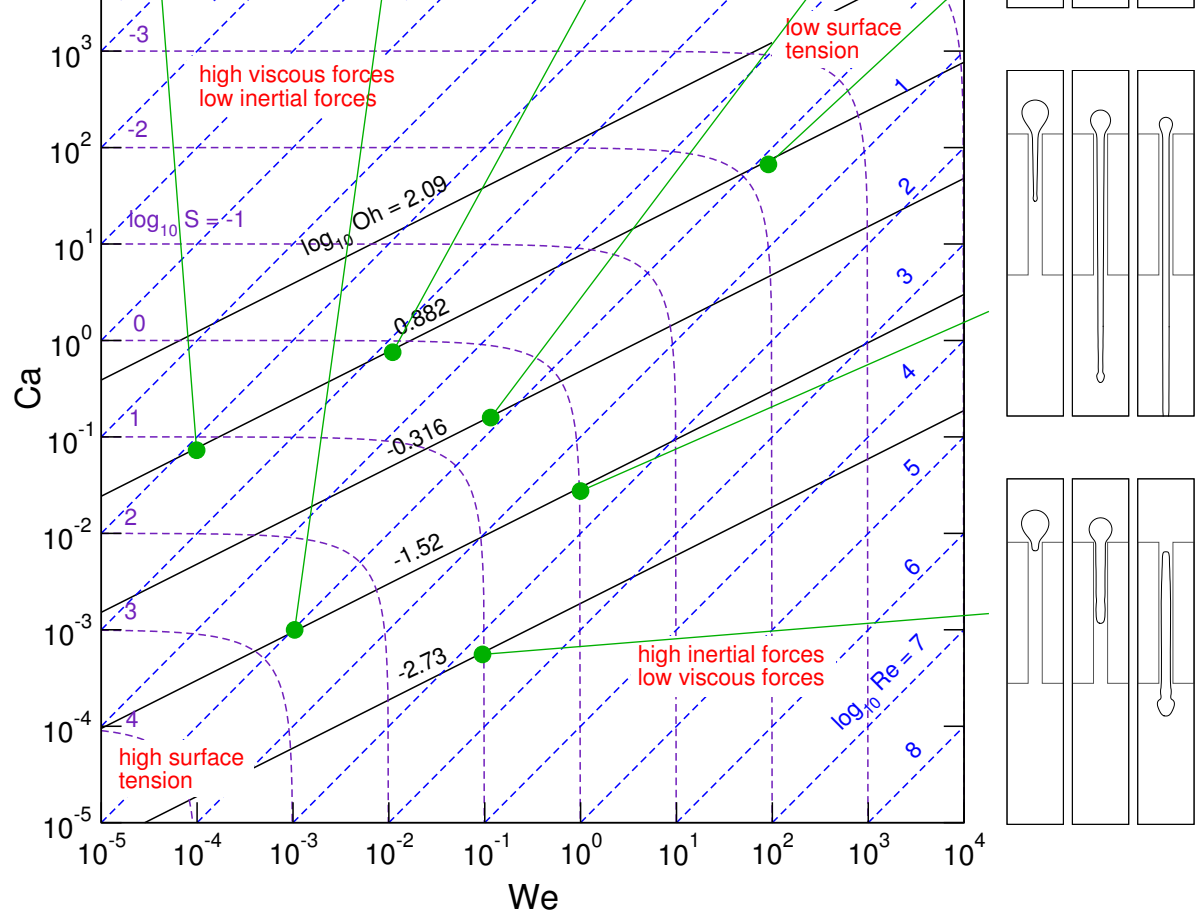

(g)

Figure 2: A phase chart of droplet deformation behaviour for $\mu_{\mathrm{d}}=1$. The dimensionless groups and displayed times for each case are: (a) $\operatorname{Re}=$ $2.13 \times 10^{-3}, S=2.13 \times 10^{1}, t=0.24,0.48,0.60 ;(b) \operatorname{Re}=1.05 \times 10^{0}$, $S=5.13 \times 10^{2}, t=0.30,0.44,0.60 ;$ (c) $\operatorname{Re}=1.30 \times 10^{-2}, S=1.28 \times 10^{0}$, $t=0.20,0.56,0.70$; (d) $\operatorname{Re}=6.59 \times 10^{-1}, S=3.97 \times 10^{0}, t=0.24,0.58,0.70$; (e) $\operatorname{Re}=1.30 \times 10^{0}, S=5.66 \times 10^{-3}, t=0.24,0.62,0.80$; (f) $\operatorname{Re}=3.33 \times 10^{1}$, $S=9.71 \times 10^{-1}, t=0.24,0.50,0.64 ;(\mathrm{g}) \operatorname{Re}=1.69 \times 10^{2}, S=9.94 \times 10^{0}$, $t=0.26,0.40,0.60$. In each image fluid flows from top to bottom. 
flow rates.

Figure 2e shows the results of a simulation conducted with a moderate value of Re but low $S$. The low value of $S$ implies that surface tension forces have only a small effect on the deformation of this droplet. A characteristic feature of this simulation is the forked tail that the droplet develops within the contraction. This tail develops because the centreline velocity within the contraction is higher than that near the walls of the contraction. This causes the interface of the droplet near the centreline to move faster through the contraction than the interface located at positions of larger $r$, resulting in the observed 'fork'. As surface tension effects are very weak here, the droplet does not return to the form of a sphere after exiting the contraction.

Figure 2c shows a simulation conducted with a lower value of Re to that shown in Figure 2e, but now with a moderate value of $S$. The higher surface tension forces here act to smooth interface regions of high curvature. As a result, the tail that the previous droplet developed within the contraction is smaller, and after the droplet leaves the contraction, it assumes a less elongated shape than observed previously. Figures $2 \mathrm{~d}, 2 \mathrm{a}$ and $2 \mathrm{~b}$ show that as $S$ is increased, the droplet shapes become smoother and fatter within the contraction, and return to more spherical shapes after they exit. In Figure $2 \mathrm{~b}$ surface tension effects are so great that significant shape changes only occur when the droplet is constrained by the contraction walls. Thus, within the contraction the droplet forms a thick capsule having approximately the same radius of the contraction, while beyond the contraction, it quickly reforms back to its initial spherical state. The difference in Re between these five cases (Figures 2a-e) appears to have little effect on the observed droplet shapes, implying that below $\mathrm{Re} \approx 1$ the inertial effects are small.

Two higher Re cases are shown in Figure 2. Figure $2 \mathrm{f}$ shows a case with a slightly lower value for $S$ than in Figure 2c, but now with a substantially higher Re. The larger inertial effects experienced in this case result in a strong jet of fluid emerging from the contraction, and this jet drives large recirculation zones that extend beyond the computational boundaries. 
As the recirculation zones extend beyond the computational domain, their behaviour is not reliably predicted. However, tests using a variety of computational domain sizes show that the shape of these recirculation zones does not significantly affect droplet deformation here, most probably because the droplet does not enter these zones during the simulation.

The larger inertial effects present in Figure $2 \mathrm{f}$ compared with those in Figure $2 \mathrm{c}$ mean that the droplet requires a longer time to accelerate at the entrance to the contraction. However, once within the contraction the droplet forms a filament of fluid whose leading tip moves at approximately the contraction centreline velocity, significantly faster than observed in the lower Re cases. As the inertia of this filament is high, its shape remains substantially intact after leaving the contraction. Note that the diameters of the filaments formed within the contraction are similar in cases $2 \mathrm{f}$ and $2 \mathrm{c}$.

A characteristic feature of Figure $2 \mathrm{f}$ is the bulging of the leading tip of the droplet as it moves through the domain. This bulging is the result of surface tension forces which increase the pressure inside the high curvature region at the tip of the thread, in turn pulling this tip back towards the main body of the droplet.

Figure $2 \mathrm{~g}$ has a similar $S$ value to Figure 2a, but a substantially higher Re value. Although surface tension forces are strong in both cases, the higher inertial forces present in Figure $2 \mathrm{~g}$ mean that the droplet takes longer to accelerate at the entrance to the contraction, and the leading tip of the droplet moves some distance beyond the exit of the contraction before substantial 'tip bulging' occurs. However, the width of the filament within the contraction is similar in both cases.

\section{Results: lower viscosity droplet, $\mu_{\mathrm{d}}=0.1$}

A second series of simulations was conducted with a smaller disperse continuous phase viscosity ratio $\left(\mu_{\mathrm{d}}=0.1\right)$. Selected results from these 
5 Results: lower viscosity droplet, $\mu_{\mathrm{d}}=0.1$
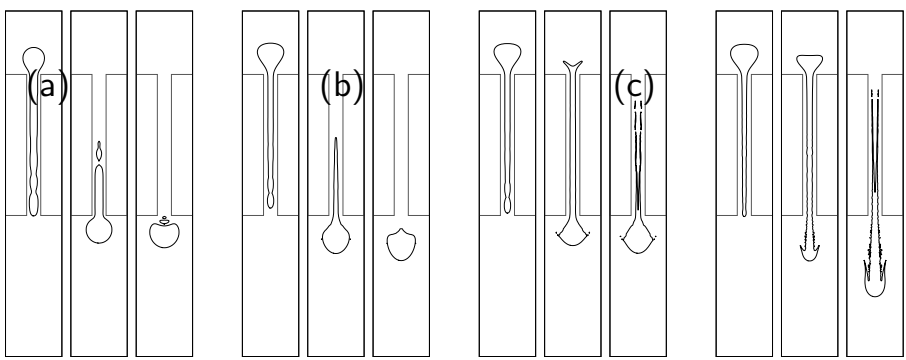

(d)
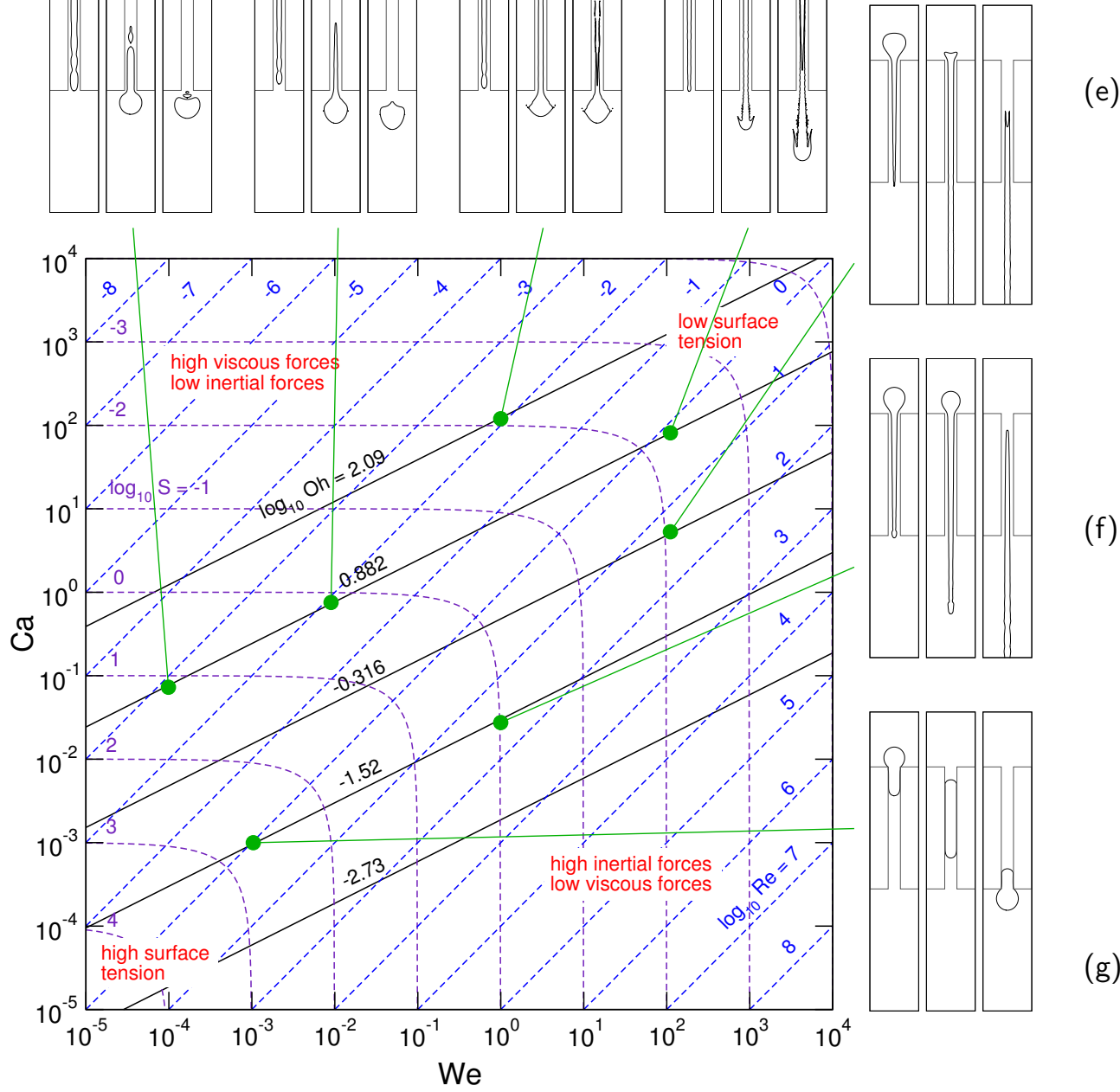

(g)

Figure 3: A phase chart of droplet deformation behaviour for $\mu_{\mathrm{d}}=0.1$. The dimensionless groups and displayed times for each case are: (a) $\mathrm{Re}=$ $2.13 \times 10^{-3}, S=2.13 \times 10^{1}, t=0.22,0.42,0.50$; (b) $R e=1.30 \times 10^{-2}$, $S=1.28 \times 10^{0}, t=0.18,0.58,0.70$; (c) $\operatorname{Re}=8.14 \times 10^{-3}, S=8.06 \times 10^{-3}$, $t=0.18,0.46,0.58$; (d) $\operatorname{Re}=1.30 \times 10^{0}, S=5.66 \times 10^{-3}, t=0.22,0.40,0.66$; (e) $\operatorname{Re}=2.08 \times 10^{1}, S=9.54 \times 10^{-3}, t=0.28,0.68,0.82$; (f) $\operatorname{Re}=3.33 \times 10^{1}$, $S=9.71 \times 10^{-1}, t=0.34,0.46,0.80 ;(\mathrm{g}) \operatorname{Re}=1.05 \times 10^{0}, S=5.13 \times 10^{2}$, $t=0.13,0.40,0.60$. In each image fluid flows from top to bottom. 


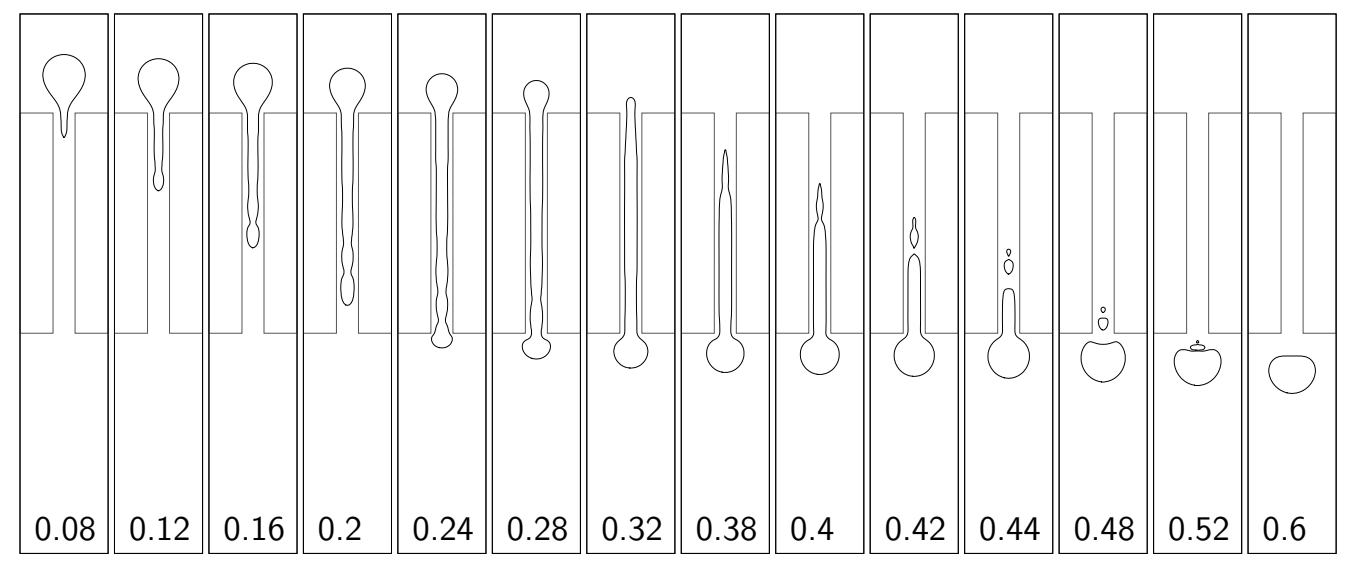

FiguRE 4: Images from the simulation of Figure 3a shown at indicated times. Here $\operatorname{Re}=2.13 \times 10^{-3}, S=2.13 \times 10^{1}$ and $\mu_{\mathrm{d}}=0.1$. In each image fluid flows from top to bottom.

simulations are shown in Figure 3, along with a corresponding Ca verses We phase chart.

Figure 3a shows images from a simulation conducted with a high level of surface tension $S$, but low Re. A series of time steps from this simulation are shown in Figure 4. Three interesting features of this simulation are: the significant bulging of the leading tip as it moves through the contraction, the growth of capillary waves along the filament when it is within the contraction, and the shedding of droplets from the rear of the droplet as it exits the contraction.

The formation of a 'bulge' at the leading tip of the droplet between times 0.12 and 0.24 in Figure 4, and at the receding tip of the same droplet between times 0.38 and 0.44 , is due to the same physical mechanism that produced the tip bulging seen in Figure 2f. Surface tension forces increase the pressure within the disperse phase behind the high curvature tip. This forces fluid away from the tip and back towards the bulk of the droplet, in turn increasing 
the radius of the tip and producing the bulge.

The behaviour of these tips has much in common with the behaviour of the tip of a relaxing extended droplet, as studied by Powers et al. [7] and Stone \& Leal [10] for low inertia flows. Stone \& Leal propose that the stability of the bulge at the tip of a receding extended droplet (that is, whether the tip will 'pinch-off' from the rest of the droplet or not) is determined by the competition between two forces. One force is a drag force acting on the bulge of the tip by the surrounding fluid. For Stone \& Leal's geometry where the bulge is moving towards the bulk of the droplet in an otherwise quiescent fluid, this force is directed away from the bulk of the droplet and tends to promote 'pinch-off' of the tip from the rest of the droplet. Another force, due to surface tension on the droplet side of the bulge, acts to pull the bulge back towards the bulk of the droplet, thus suppressing pinch-off. If this pulling force is less than the drag force, then a neck has time to form adjacent to the bulge, eventually severing the tip from the remainder of the droplet and producing a satellite droplet. Conversely, if the drag force is less than the surface tension force, then there is insufficient time for a neck to form next to the bulge and the tip will recede in a steady manner back along the length of the filament.

This type of analysis, applied to the leading and rear tips of the droplet shown in Figure 4, explains why the leading tip of the droplet is stable in our example while the rear tip of the droplet sheds small droplets. At the leading tip of the droplet, the velocity of the tip is higher than that of the surrounding continuous phase. As a result, the drag force acting on the leading bulge is directed towards the bulk of the droplet and acts to stabilise this bulge. However, at the rear of the droplet the situation is reversed. Here the continuous phase velocity is moving away from the tip of the droplet (relative to the droplet tip) so that the drag force on the rear bulge is directed away from the bulk of the droplet. This situation is similar to that examined by Stone \& Lea [10] and explains why small droplets are shed from the rear of the filament in this case. Numerical simulations performed by Powers et 
al. [7, see Figure 7] suggest that the time between pinch-off for a droplet having the dimensions of the filament shown in Figure 4 but in a quiescent fluid would be around 0.14 non-dimensional time units. This appears to be just a little longer than the time between when the rear of the droplet enters the contraction and the first pinch-off occurs in our simulations. However, this is consistent with the drag force being slightly higher in our geometry than in Powers et al.'s otherwise quiescent fluid.

The simulations predict that the small satellite droplets shed from the rear tip of the filament merge again with the bulk of the droplet as it exits the contraction (see times 0.52 and 0.6 in Figure 4). The satellite droplets approach the main droplet at the exit to the contraction as the centreline velocity decelerates here. However, whether or not real droplets would coalesce in this situation cannot be predicted by the VOF method as the dynamics of the thin film existing between the interfaces of the droplets cannot be resolved on the spatial scale of the computational mesh. Thus, the coalescence of the satellite droplets observed in Figure 4 may or may not be physical.

Figure 4 also shows the development of capillary waves along the filament surface when it is in the contraction, particularly behind the leading tip bulge. The creeping flow linear stability analysis of Tomotika [11] suggests that the fastest growing wavelength for capillary instabilities along a filament of this size would be around 1.37, with a corresponding growth rate of 13.0 (that is, it would take 0.177 non-dimensional time units for the amplitude of a disturbance to increase by an order of magnitude). This wavelength and growth rate are consistent with the results of the figure, suggesting that capillary waves are producing the radial oscillations observed in this simulation.

Figure $3 \mathrm{~b}$ shows a simulation conducted with a smaller level of $S$, but still with a low Re. In this case the leading bulge is smaller than in Figure 3a, and no droplets are shed from the rear tip during the deformation. These results are consistent with the analysis of Powers et al. [7] who predict a pinch-off time for a filament of this size of around 1.86, which is significantly larger than the time the filament spends in the contraction. Also, there is 
less capillary wave growth along the extended filament in this example than in Figure 3a. The linear stability analysis of Tomotika [11] gives a maximum growth rate for capillary waves along a filament of this size of 0.941 . This growth rate is significantly slower than the growth rate calculated for the example of Figure 3a (the growth rate scales with $\mathrm{Ca}^{-1}$ ), thus, the filament does not exist within the contraction for enough time for interface oscillations to develop.

Figure 3c shows a simulation where $S$ has been decreased further while Figure $3 \mathrm{~g}$ shows a simulation where $S$ is very high. Like the higher viscosity simulation shown in Figure 2e, the surface tension forces in the simulation shown in Figure 3c are small and a fork develops at the rear tip of the droplet. The case shown in Figure $3 \mathrm{~g}$ is very similar to that shown in Figure $2 \mathrm{~b}$ - here $S$ is so large that deformation only occurs when the droplet is constrained by the contraction walls.

The simulations shown in Figures 3d, e and $\mathrm{f}$ show the effect of increasing inertia on droplet deformation. Similar to the results shown in Figures $2 \mathrm{f}$ and $g$, as Re is increased, inertial forces cause the droplet to accelerate at a slower rate when entering the contraction, and to 'jet' when exiting the contraction.

The example of Figure 3d, and to a lesser extent the examples in Figures $3 \mathrm{c}$ and e, show the development of small wavelength instabilities along the filament surface, especially within the lower half of the contraction. These waves are particularly apparent in Figure $3 \mathrm{~d}$ where they shorten and grow radially as the filament decelerates and expands when leaving the contraction.

Linear stability results presented in Lee \& Flumerfelt [6] for moderate Re capillary wave growth suggests that for the example shown in Figure 3d, a wavelength of around 1.11 has the largest possible growth rate. Clearly this length is larger than the wavelengths observed along the filament in the simulations, suggesting that the instabilities observed in the figure are not 
surface tension related. It is more likely that the source of these waves are small wavelength instabilities that can occur at a discrete interface in the absence of surface tension. Such instabilities are similar in nature to KelvinHelmholtz instabilities, and have been described analytically by Hooper \& Boyd [5].

\section{Conclusions}

The deformation of droplets as they pass through an axisymmetric microfluidic contraction has been simulated over a range of Re and $S$, and for two disperse continuous phase viscosity ratios. A variety of shapes were observed during the simulations, ranging from thick slugs through to thin filaments. In some of the lower viscosity disperse phase simulations instabilities grew along the surface of the extended droplets. In one particular case this lead to droplet breakup within the contraction. Future work will extend the analysis to different viscosity ratios and to non-Newtonian fluids.

Acknowledgment: This research was supported by the Australian Research Council Linkage Grants Scheme.

\section{References}

[1] S. L. Anna, N. Bontoux, and H. A. Stone. Formation of dispersions using 'flow focusing' in microchannels. Applied Physics Letters, 82(3):364-366, Jan 20 2002. C152, C155

[2] M. R. Davidson. VOF prediction of drop formation of shear-thinning and yield stress fluids. In 5th International Conference on Multiphase Flow, ICMF'04, Yokohama, Japan, May 30-June 4 2004. Paper No. 501. $\mathrm{C} 154$ 
[3] J. Eggers. Nonlinear dynamics and breakup of free-surface flows. Reviews of Modern Physics, 69(3):865-929, Jul 1997. C152

[4] C. D. Han and K. Funatsu. An experimental study of droplet deformation and breakup in pressure-driven flows through converging and uniform channels. Journal of Rheology, 22(2):113-133, 1978. C152

[5] A. P. Hooper and W. G. C. Boyd. Shear-flow instability at the interface between two viscous fluids. Journal of Fluid Mechanics, 128:507-528, 1983. C164

[6] W.-K. Lee and R. W. Flumerfelt. Instability of stationary and uniformly moving cylindrical fluid bodies - i. International Journal of Multiphase Flow, 7:363-383, 1981. C163

[7] T. R. Powers, D. Zhang, R. E. Goldstein, and H. A. Stone. Propagation of a topological transition: The rayleigh instability. Physics of Fluids, 10(5):1052-1057, 1998. C161, C162

[8] M. Rudman. A volume-tracking method for incompressible multifluid flows with large density variations. International Journal for Numerical Methods in Fluids, 28:357-378, 1998. C154

[9] H. A. Stone. Dynamics of drop deformation and breakup in viscous fluids. Annual Review of Fluid Mechanics, 26:65-102, 1994. C152

[10] H. A. Stone and L. G. Leal. Relaxation and breakup of an initially extended drop in an otherwise quiescent fluid. Journal of Fluid Mechanics, 198:399-427, 1989. C161

[11] S. Tomotika. On the instability of a cylindrical thread of a viscous liquid surrounded by another viscous fluid. Proceedings of the Royal Society of London, Series A, 150(870):322-337, June 1935. C162, C163

[12] D. S. Whyte, J. Cooper-White, M. Davidson, A. Lunqvist, and P. Schaerringer. Deformation of a droplet passing through a 
contraction. In Proceedings of FEDSM2002: 2002 ASME Fluids Engineering Division Summer Meeting, Montreal, Quebec, Canada, July 14-18 2002. ASME. C152, C154

[13] D. L. Youngs. Time-dependent multimaterial flow with large fluid distortion. In K. Morton and M. Baines, editors, Numerical Methods for Fluid Dynamics, pages 273-285. Academic Press, 1982. C154 